\title{
AN EXPERIMENTAL STUDY ON FIGURAL AFTER- EFFECT BY THE MEASUREMENT OF FIELD STRENGTH
}

\author{
SHIN NOZAWA \\ Hokkaido University
}

\section{PROBLEM}

Recently, Yokose $(\mathbf{1 0}, \mathbf{1 1})$ has measured the strength of influence that a figure has upon its field by measuring the threshold of a small light spot presented around the figure, and he succeeded in inducing functional formula. Several experimenters iuquired into this problem more closely by various methods and they obtained similar results $(6,7,8)$. The author, however, does not consider their methods to be the best, because of these two reasons : for (1) they neglect the time effect; (2) if presentation of a figure and measurement of the threshold of a light spot are done simultaneously, identity of the operation for each condition is not always assured. To avoid this defect, it will be better to operate them separately in two passages. If we consciously change the time for presenting stimulus figure, or the time interval between presentation and measurement, we will be able to examine the time effect more closely. The first aim of this report is to what extent such an indirect measurement as this is possible. Next, theoretically this measurement is no more than a measurement of so called figural after effect. So, if the measurement mentioned above is possi-

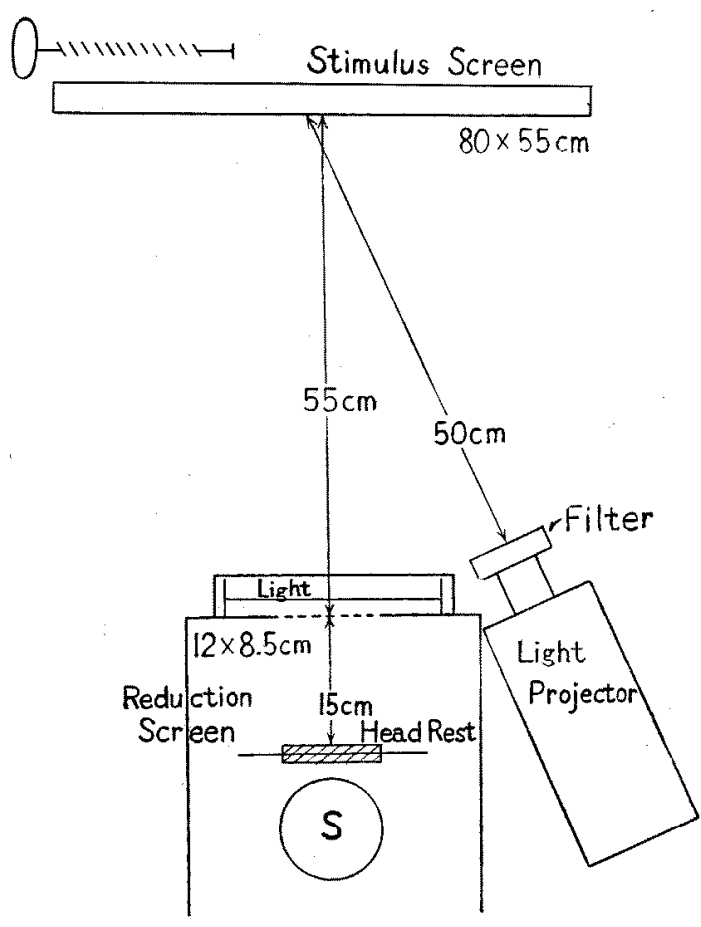

Fig. 1

ble, the result may well be of use for understanding the facts about figural 
after effect already known. This is the second aim of this report.

\section{APPARATUS}

Equipment for the investigation is set up as Fig. 1. in a darkened room.

(1) Apparatus for the presentation of stimulus figure: A vertical screen $(80 \mathrm{~cm} /$ $65 \mathrm{~cm}$ ) is set at the height of a subject's eyes. On this screen, inspection figure and test figure are hung one upon the other and fixed together by thumb tacks. Stimulus screen is illuminated indirectly from the position $50 \mathrm{~cm}$. apart. (Strength of light upon the surface of this stimulus screen is 20.25 radlux).

(2) Apparatus for the measurement of stimulus threshold: A small light spot $(0.3 \mathrm{~mm}$. in diametre) is projected on the screen $1 \mathrm{~cm}$. aside (right or left) from the fixation point which is located in the centre of the stimulus screen. Brightness of light can be adjusted continuously by a polarization-filter.

\section{(3) Apparatus for the observation:} A subject sits up at the distance of $70 \mathrm{~cm}$. from the stimulus screen, using head-rest, and observes through a reduction screen $(8.5 \mathrm{~cm} / 1.2 \mathrm{~cm}$.) located at the distance of $55 \mathrm{~cm}$. from stimulus screen.

\section{Procedure of MAIN EXPERIMENT}

The order of the measurement Test I. As the subject sits up at the seat for observation, the experimenter projects a light spot ( 33 radlux) in a given position on the stimulus screen, and then lowers the brightness of the light spot rapidly in a regular speed. S. signs as soon as the light spot becomes invisible. The experimenter records the brightness at that moment.

Prolonged inspection period The experimenter presents inspection figure on the stimulus screen. It is neccessary that the fixation point of inspection figure coincides with that of the stimulus screen. S. keeps fixing his eyes upon the fixation point for one hundred and twenty seconds.
Test II. As the prolonged inspection period is over, the expepimenter removes the inspection figure and goes on operating the measurement in the same process as that of Test I.

Amount of figural after effect The author considers that the difference of measured values between Test I and Test II indicates the amount of figural after-effect by the inspection figure, on condition that the measured values do not change to any direction by repeating test like this.

Subjects Four to six subjects consisting of students and members of Psychological laboratory in Hokkaido University were used.

\section{PRELIMINARY EXPERIMENT}

Purpose Before starting the main experiment, it is neccessary to affirm that the hypothesis mentioned in the parenthesis in the last part of the procedure, is trustworthy.

Procedure Here, inspection figure is not used but the measurement shown in Test I is performed ten times in rapid succession. The experimenter examines the trend of the result.

Results Shown in Table 1. As for the results of this experiment. Repetition of measurement did not produce any particular tendency of either inorease or decrease of measured values. (Recognized by trend test of non parametric method). Accordingly, the hypothesis may well be considered satisfactory in outline.

\section{EXPERIMENT A}

Purpose "If we present a straight line after prolonged inspection of a curved line in the same position as before, the straight line looks as if distorted to the opposite direction; while prolonged inspection of a straight line brings out no change like that." These phenomena are called Gibson's curved line effect (1). The aim of 
Table 1

Preliminaly Exp. (thrshold of light spot radlux)

\begin{tabular}{c|cccccccccc|c}
\hline Nr. of Times & 1 & 2 & 3 & 4 & 5 & 6 & 7 & 8 & 9 & 10 & A.V. \\
Ss. & & & & & & & & & \\
\hline NOZ. & 23.41 & 24.65 & 24.35 & 24.23 & 23.64 & 24.35 & 24.35 & 24.12 & 23.85 & 24.99 & 24.194 \\
NKN. & 23.07 & 27.40 & 26.42 & 26.42 & 26.06 & 27.07 & 27.75 & 26.86 & 25.11 & 26.71 & 26.687 \\
KTN. & 23.30 & 22.96 & 22.54 & 22.96 & 22.64 & 23.64 & 23.53 & 24.86 & 23.64 & 23.53 & 23.360 \\
MRY. & 24.65 & 24.48 & 25.11 & 24.75 & 25.22 & 24.48 & 25.11 & 23.64 & 24.65 & 24.65 & 24.674 \\
OGR. & 25.22 & 25.22 & 25.42 & 25.11 & 25.11 & 25.22 & 24.99 & 24.86 & 25.90 & 25.11 & 25.126 \\
\hline A.V. & 24.730 & 24.942 & 24.768 & 24.694 & 24.534 & 24.952 & 25.126 & 24.868 & 24.430 & 24.998 & 24.830
\end{tabular}

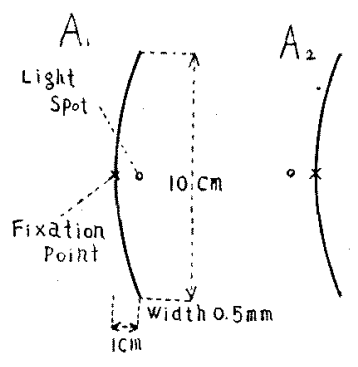

B.

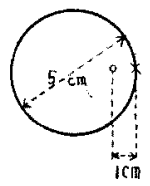

D.

$D_{2} \circ$

$\mathrm{B}_{2}$

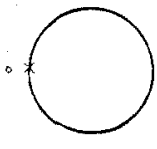

C.
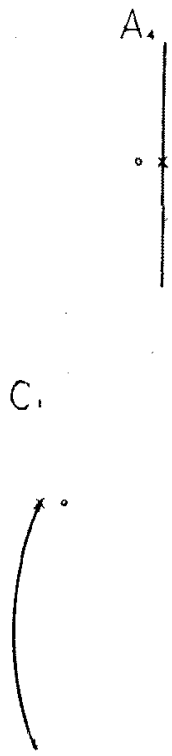

.

$\mathrm{C}_{2}$

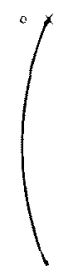<smiles>[2H][13CH][13CH]</smiles>

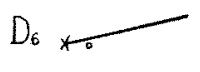

${ }^{D} \Upsilon^{\circ}$

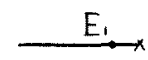

$E_{2}$

Fig. 2 
these experimental series is to measure and compare the effects which prolonged inspection of curved line and straight line has upon the threshold of a light spot.

Condition The arrangement of each stimulus figure is shown in Fig. II. A.

Exp. $A_{1}$ : Prolonged inspection of a curved line. A light spot is presented
Results Only the amount of after effect is shown in Table 2.

1) There is no significant difference among the results of Test I throughout this experimental series (In Table 2, the results of Test I are omitted.). From these results, it can be claimed that Test I was done almost in the same condition throughout this experi-

Table. 2

Exp. A

\begin{tabular}{c|ccccc}
\hline Cond. & \multicolumn{1}{c}{$\mathrm{A}_{1}$} & \multicolumn{1}{c}{$\mathrm{A}_{2}$} & $\mathrm{~A}_{3}$ & $\mathrm{~A}_{4}$ & $\mathrm{~A}_{0}$ \\
\hline Ss. & -1.56 & +3.01 & -0.40 & -0.55 & +0.23 \\
MRY. & -2.64 & +1.36 & -0.24 & +0.13 & -0.24 \\
KTN. & -0.99 & +1.69 & -0.80 & -0.66 & +0.31 \\
OGR. & -1.12 & +0.61 & -0.18 & -0.21 & -0.16 \\
TRN. & -0.55 & +0.35 & -0.57 & -0.33 & -0.71 \\
TGW. & $(-1.81)$ & $(+3.52)$ & $\sim$ & $\sim$ & $\sim$ \\
NKN. & -1.372 & +1.405 & -0.438 & -0.324 & -0.114
\end{tabular}

on the right side of the fixation point (inside of the curved line).

Exp. $\mathrm{A}_{2}$ : Prolonged inspection of a curved line. A light spot is presented on the left side of the fixation point (outside of the curved line).

Exp. $A_{3}$ : Prolonged inspection of a straight line. A light spot is presented on the right side of the fixation point.

Exp. $A_{4}$ : Prolonged inspection of a straight line. A light spot is presented on the left side of the fixation point.

Exp. $A_{0}$ : Prolonged inspection of only the fixation point. A light spot is presented on the right side. mental series. The position of light spot, whether it may be located on the right or the left side of the fixation point, produced no distinct difference in the threshold.

2) There is no significant difference between the results of Test I and II in Exp. $A_{0}$. This proves that prolonged inspection of nothing but the fixation point gave no considerable effect in Test II. Exp. $\mathrm{A}_{0}$ serves as the control experiment for the other experiments.

3 ) In Exp. $A_{3}$ and $A_{4}$, the difference between the results of Test I and II is statistically significant. (Level of sig. nificance $\alpha<0.05)$. Prolonged inspec- 
tion of a straight line produced higher threshold in Test II than in Test I.

4) There is no significant difference between the results of Exp. $A_{3}$ and Exp. $A_{4}$. This means that the effect of the inspection figure shown in (3) is nearly the same on both sides.

5) The amount of Exp. $A_{1}$ is distinctly greater than those of Exp. $A_{3}$, and $\mathrm{A}_{4} \cdot(\alpha<0.05)$. It can be said that the influence of inspection figure is far greater inside a curved line on either side of a straight line, if they are of the same length.

6) There is an apparent after-effect in Exp. $A_{2}$. also. However best care should be taken to see that the direction of influence is opposite to the previous one. That is, the stimulus threshold of light spot, if placed outside the curved line, decreases after the prolonged inspection.

7) Seeing through the results above, it becomes apparent that, by an indirect way of measuring the stimulus threshold of a light spot, we might be able to examine the effect of inspection figure.

8) It is interesting to see the fact that there is no difference of the effect on both sides of the vertical line, while, on both sides of the curved line, there is a remarkable difference. And comparison of these with so-called Gibson effect is especially inviting for the author.

\section{EXPERIMENT B}

Purpose "When a contour figurefor example, a circle,--is seen under the prolonged inspection, its phenomenal size shrinks gradually. This effect can be proved by the test figure, coincidental with the inspection figure. (Köhler's size effect) $(1,5) . "$ The aim of this Experimental series $B$ is to measurethe effect of a circle under the prolonged inspection in this case, by measuring the stimulus threshold of a light spot which is presented on either side of the contour. And also, to compare the one with the other.

Condition Exp. $\mathrm{B}_{1}$ : Prolonged inspection of a circle. A light spot is presented on the left side of the fixation point. (inside).

Table. 3

\begin{tabular}{c|cc}
\multicolumn{3}{|c}{ Exp. B } \\
\hline Cond. & $\mathrm{B}_{1}$ & $\mathrm{~B}_{2}$ \\
\hline Ss & -2.13 & +1.96 \\
MRY. & -0.57 & +2.26 \\
OGR. & -1.66 & +0.51 \\
TRN. & -0.64 & +1.94 \\
NKN. & -1.00 & +1.334 \\
A.V. & &
\end{tabular}

Exp. $B_{2}$ : Prolonged inspection of a circle. A light spot is presented on the right side of the fixation point. (outside). (Fig. II. B)

Results Shown in Table 3. 1) As for Exp. $B_{1}$, there is evident after-effect $(\alpha<0.05)$. The result is the same as in the case of Exp. $A_{1}$ : Namely, the 
stimulus threshold is higher in Test II than in Test $I$, because of the influence of inspection figure. (Hereafter, the author will call this - effect, for short.)

2) As for Exp. $B_{2}$, remarkable aftereffect is recognized. $(\alpha<0.05)$ The result of this being the same as of Exp. $A_{2}$, the stimulus threshold is lower, in Test II by the influence of inspection figure, than in Test I. (For short, let us call it +effect.)

3) As for the contour line of circle, there can be recognized difference of the effect, between inside and outside just like the one observed between both sides of the curved line in Exp. $A_{1}$ and $\mathrm{A}_{2}$.

\section{EXPERIMENT C}

The author's second aim is to inquire into the problem of figural after effect such as Gibson's curved line effect, or, Köhlers's size effect, by the help of the result of this new measurement.

The result of Exp. $A_{1}$. and $A_{2}$ referring Gibson's effect and that of Exp. $B_{1}$ and $B_{2}$ referring Köhler effect seem to coincide with each other. That is, as for curved line, or contour line, after effect, observed inside the line is recognized as -effect; and that outside, as +effect. Why, then, do these effects cause the decrease of curvature in case of a curved line, while the same effects cause the increase of curvature in case of a circle? To solve this problem, it is necessary to measure the influence around the tip of the curved line (not only near the centre).

Purpose This is to examine the effect of prolonged inspection of a curved line by measuring the stimulus threshold of a light spot located near the upper end of the line.

Condition Exp. $\mathrm{C}_{1}$ : Prolonged inspection of a curved line. A light spot is presented on the right side of the fixation point (inside the upper end of the curved line.)

Exp. $C_{2}$ : Prolonged inspection of a curved line. A lignt spot is presented on the left side of the fixation point. (outside the upper end of the curved line.) (Fig. 2. C.)

Table. 4

Exp.C

\begin{tabular}{c|cc}
\hline Cond. & $\mathrm{C}_{2}$ & $\mathrm{C}_{2}$ \\
\hline Ss & +1.12 & -0.16 \\
OGR. & +0.50 & -1.62 \\
TRN. & +2.84 & -1.64 \\
KTN. & +2.52 \\
MTH. & +0.72 & -0.52 \\
MRY. & +0.59 & -0.11 \\
NKN. & +1.22 & -0.53 \\
\hline A.V. & +1.161 & -0.763
\end{tabular}

Results Shown in Table 4. 1) In either Exp. $C_{1}$ and Exp. $C_{2}$, there is a clear difference to be recognized. ( $\alpha<$ 0.05 ).

2) The direction of the after effect in Exp.C is entirely opposite to that in Exp. A : That is, in Exp. C the after effect is -effect outside the curved line, and +effect, when inside. 
3) Referring Exp. A B C, when the direction of displacement and the differencc of after effect measured on both sides of a line are put altogether into consideration, it can be said that displacement always occurs from the position where +effect is observed to the position where - effect is observed. (Fig. III).
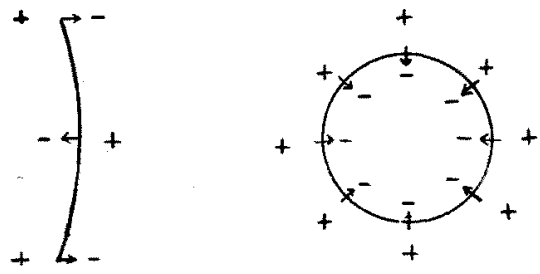

Fig. 3

\section{EXPERIMENT D}

Purpose We examine the hypothesis shown in Fig. III, whether it should be appropriate or not, for such phenomenon as "titled line effect" by Gibson. (Titled line under prolonged inspection has a tendency to approach by degrees the norm. (2.3) In this case, titled line being nearly horizontal becomes horizontal and, the line nearly vertical, becomes vertical.)

Condition Exp. $\mathrm{D}_{1}$ : Prolonged inspection of $10^{\circ}$ tilted line. A light spot is presented on the right side of the fixation point. (the lower central part of the tilted line).

Exp. $D_{2}$ : Prolonged inspection of $10^{\circ}$ tilted line. A light spot is presented on the left side of the fixation point. (the upper central part of the tilted line.)
Exp. $\mathrm{D}_{3}$ : Prolonged inspection of $10^{\circ}$ tilted line. A light spot is presented on the right side of the fixation point. (the lower side of the upper end of the tilted line.)

Exp. $\mathrm{D}_{4}$ : Prolonged inspection of $10^{\circ}$ tilted line. A light spot is presented on the left side of the fixation point. (the upper side of the upper end of the tilted line.)

Exp. $D_{5}$ : Prolonged inspection of $80^{\circ}$ tilted line. A light spot is presented on the left side of the fixation point. (the upper side of the tilted line.)

Exp. $\mathrm{D}_{\sigma}$ : Prolonged inspection of $80^{\circ}$ tilted line. A light spot is presented on the right side of the fixation point. (the lower side of the tilted line.)

Exp. $D_{7}:$ Prolonged inspection of vertical line $\left(0^{\circ}\right)$. A light spot is presented on the right side of the fixation point. (the right side of the upper end of the vertical line.) Fig. II. D.

Results Shown in Table 5. 1) There is recognized apparent after effect in every condition.

2 ) In Exp. $D_{2}$. and $D_{2}$, (quality of the) after effects are altogether - effect. It can not be said that there is significant difference in the amount of after effect between them. However, it seems that these influences in the field do not affect much the diplacement of tilted line.

3.) In Exp. $\tilde{D}_{3}$, there can be recognized +effect. And in $D_{4}$-effect is seen. According to our hypothesis, tilted line, in such situation, should 
displace counter clockwise, that is, to approach the vertical. is presented at the right side of the fixation point (on the extension of the

Table. 5

Exp. D

\begin{tabular}{|c|c|c|c|c|c|c|c|}
\hline $\begin{array}{l}\text { Cond. } \\
\text { Ss }\end{array}$ & $\mathrm{D}_{1}$ & $\mathrm{D}_{2}$ & $D_{3}$ & $D_{4}$ & $D_{i}$ & $D_{6}$ & $\Sigma_{7}$ \\
\hline OGR. & -0.46 & -0.29 & +0.95 & -0.83 & +1.25 & -1.33 & +0.48 \\
\hline MTH. & -0.16 & -0.42 & +0.64 & -0.42 & +1.01 & -0.48 & +0.32 \\
\hline TGW. & -0.23 & -0.87 & +1.45 & -0.59 & +2.16 & -3.28 & +0.94 \\
\hline TRN. & -0.18 & -1.11 & +0.52 & -1.16 & +0.91 & -0.16 & +0.66 \\
\hline A. V. & -0.053 & -0.673 & +0.890 & $-0.75 \mathrm{~J}$ & +1.333 & -1.273 & +0.60 \\
\hline
\end{tabular}

3) In Exp. $D_{\tilde{j}}$, there is recognized + effect, and in $D_{6}$, - effect is seen. According to our hypothesis tilted line, in such situation, should displace clockwise, namely, approach the horizontal.

5) In Exp. $D_{\eta}$, + éffect was obtained. The experiment in this condition was done as a control. It is interesting to compare this with those of Exp. $A_{3} A_{4}$; or with Exp. $D_{3} D_{4}$.

\section{EXPERIMENT E}

Purpose It has been known that the straight line, under prolonged inspection, does not change in shape but in length, as if it were shortened. The aim of Exp. E. is to test this kind of effect.

Condition Exp. $\mathrm{E}_{1}$ Prolonged inspection of a horizontal straight line. A light spot is presented on the left side of the fixation point (on the straight line).

Exp. $\mathrm{E}_{2}$. Prolonged inspection of a horizontal straight line. A light spot
Table. 6

Exp. E

\begin{tabular}{c|cc}
\hline Cond. & $\mathrm{E}_{1}$ & $\mathrm{E}_{2}$ \\
\hline Ss & & -1.01 \\
\hline OGR. & -2.79 & -1.08 \\
MTH. & -1.52 & -1.08 \\
TGW. & -1.72 & -0.91 \\
TRN. & -2.05 & -1.34 \\
\hline A.V. & -2.030 & -1.085
\end{tabular}

straight line). (Fig. II. E.)

Results Shown in Table 6, 1) Apparent - effect $(\alpha<0.05)$ is recognized in both Exp. $E_{1}$ and Exp. $E_{2}$.

2) The amount of after effect of Exp. $E_{1}$ is more - effect than that of exp. $E_{2} . \quad(\alpha<0.05)$.

$3)$ As there is pretty amount of - effect recognized in Exp. $E_{2}$, if we see nothing but the result itself, it is hard to explain the shortening of straight line by our previous hypothesis. Because in Exp. ABC and D, whenever the displacement of test figure occured, 
there always existed two opposite effects : + effect and - effect ; however, in Exp. E, there existed only - effect. But if we admit following extension on the previous hypothesis, we may well explain them including the result of Exp. E. That is, "whether the effects obtained from these two points are both +effect or they are both - effect, if there exist any difference between the amount of effects measured on both sides of test figure, the test figure should displace in the direction of gradient of the amount of aftereffect; namely, it displaces from the point of greater amount of +effect to that of less amount of +effect; or, from the point of less amount of - effect to that of greater amount of - effect."

\section{SUMMARY AND CONCLUSIONS}

Admiting the fact that under prolonged inspection a figure affects the field around itself and the effect remains for a while after the figure is removed away, and then disappears gradually, we are able to confirm this kind of effect by comparing the stimulus threshold of a light spot (Test II) which is measured after removing the figure away, with that when there is no figure. (Test I).

2 ) If we put quality and quantity of after effect of straight line ; of curved line; and of circle, and, displacement effect of test figure hitherto known as FIGURAL AFTER EFFECT, together, we may say as follows : if after effect measured on both sides of test figure are equivalent, displacement of test figure does not occur at all, and, if there is any difference between the amount of after effect on both sides of the test line, there occurs displacement of test figure.

3) The direction of displacement of test figure coincides with the direction of gradient of after effect which was measured by light spot.

4) The result above mentioned was confirmed by both Gibson's CURVED LINE EFFECT and TILTED LINE EFFECT, and Köhler's SIZE EFFECT AND SHORTENED EFFECT OF STRAIGHT LINE.

5 ) However, as the study still remains to be a preliminary one, and the control of conditions of each experimental series is not yet satisfactory, it is absolutely necessary to go into further investigation by more systematic method.

\section{REFERENCES}

1) Gibson, J. J. : Adaptation, after-effect and contrast in the perception of curved lines. J. Exp. Psychol., 1933, 16, 1-31.

2) Gibson, J. J. and Radner, M. : Adaptation, after-effect and contrast in the perception of tilted lines I. J.Exp. Psychol., 1937, 20, $453-467$.

3) Gibson, J. J. : Adaptation, after-effect and contrast in the perception of tilted lines II. J. Exp. Psychol., 1937, 20, 553--569. 4) Köhler, W. : Dynamics in Psychology, N. Y. : Liveright Co., 1940.

5) Köhler, W. and Wallach, H. : Figural after-effect ; an investigation of visual process. Proc. Amer. Phil. Soc., 1944, 88, 269 - 357.

6) Motokawa, K. : Physiological basis of sensation, Kagaku., 1948, 18,526-537. 
7) Nozawa, S. : On the figural after-effect by intermittent presentation of inspection figure., Jap. Psychol. Research., 1955, 2, 9-16. 8) Kaneko, T. and Obonai, T.: Factors of intensity, quantity and distance in psychophysiological induction. Jap.J. Psychol., 1952, 23, 73-79.

9) Ohkawa, N.: The effect of Various figures upon C.F.F. of a flickering small patch. Jap. Psychol. Research., 1954, 1,34-44.
10) Yokose, Z. and Uchiyama, M.: The measurement of the field forces in visual perception. Jap. J. Psychol., 1952, 22, 41-56. 11) Yokose, $Z$. : "The law of the field" in visual form perception ( 1 )-A theoretical formula to seek the field strength of the form and its experimental proof-. Jap. Psychol.Research., 1954, 1, 55-64. 